Communications in Physics, Vol. 25, No. 3 (2015), pp. 195-201

DOI:10.15625/0868-3166/25/3/6994

\title{
ENHANCEMENT OF LASING EMISSION IN THE METALLIC-COATED MICROSPHERE CAVITY BASED ON Er-DOPED SILICA GLASSES
}

\author{
PHAM VAN HOI, NGUYEN THE ANH, AND NGUYEN THI THU TRANG \\ Institute of Materials Science, Vietnam Academy of Science and Technology, \\ 18 Hoang Quoc Viet Rd., Cau Giay Dist., Hanoi, Vietnam \\ LE HUU THANG \\ SMEDEC1, STAMEQ 8 Hoang Quoc Viet Rd. Cau Giay Dist., Hanoi, Vietnam \\ NGUYEN VAN AN \\ College of Sciences, Hue University, 77 Nguyen Hue str., Hue city, Vietnam
}

E-mail: hoipv@ims.vast.ac.vn

Received 27 August 2015

Accepted for publication 30 September 2015

\begin{abstract}
In this paper, we present a review of enhancement and wavelength shift of abnormal upconversion green light emission from erbium ions doped in silica with narrow linewidth in the metallic-coated microsphere cavity. Although the phenomenon of the $537 \mathrm{~nm}$ - green light emission is not yet understood, its enhancement of several orders of magnitude seems to be due to the excitation of plasmons. The metallic-coated microsphere cavity has led to the lowest threshold so far observed in microsphere cavity lasers from $0.2 \mathrm{~mW}$ to $60 \mathrm{nW}$. By combining microcavity and surface plasmonic enhancement effect the researchers have created a new kind of photonic devices.
\end{abstract}

Keywords: microcavity, upconversion emission, Er-doped silica glass, surface plasmonics.

\section{INTRODUCTION}

The conversion of infrared light to visible light through energy upconversion in erbiumdoped materials has increased considerably with the applications of compact visible sources in various fields [1-6]. Er-doped silica glass is used commercially in optical fibre amplifiers, but it has limitation by small optical cross section of the Er transitions and long radiative lifetime. The Er-doped silica microcavities can overcome these limitations. In microcavity the photon is confined in the region where the Er-ions are embedded. When the confined photon is in resonance with the Er transition, the emission is enhanced in the direction of confinement and the lifetime is decreased [7]. In the literature on microcavities with weak confining structure there was an irreversible decay of the Er excited state through spontaneous emission with large linewidth of several nanometers, but in strong light confining structure the radiative decay is a reversible process leading to strong light-matter interaction [8]. Under the strong confining microcavity, the 
emission wavelength and radiative lifetime become properties of the combined atom-cavity system and can be controlled externally [9]. The upconversion green emission of Er-ions doping into the various glasses such as silica, chalcogenide, chloride, bromide, and iodide glasses based on two transitions ${ }^{2} \mathrm{H}_{11 / 2} \rightarrow{ }^{4} \mathrm{I}_{15 / 2}$ (at around wavelength of $525 \mathrm{~nm}$ ) and ${ }^{4} \mathrm{~S}_{3 / 2} \rightarrow{ }^{4} \mathrm{I}_{15 / 2}$ (at around wavelength of $550 \mathrm{~nm}$ ), but green emission from Er-doped GaN have the wavelengths of $537 \mathrm{~nm}$ and $558 \mathrm{~nm}[1,10]$. The green laser of Er-doped glass microcavity takes transition between Stark sublevels of ${ }^{4} \mathrm{~S}_{3 / 2}$ (highest lasing level) and of the ground state ${ }^{4} \mathrm{I}_{15 / 2}$ at wavelength of $550 \mathrm{~nm}$, because the decay time of upper Stark levels of ${ }^{4} \mathrm{~F}_{7 / 2},{ }^{2} \mathrm{H}_{11 / 2}$ is very short in comparison with ${ }^{4} S_{3 / 2}$ and the inversion can be achieved between this one and ground state ${ }^{4} \mathrm{I}_{15 / 2}$. In our previous work [11] we had been shown the experimental results of narrow linewidth upconversion emission at wavelength of $537 \mathrm{~nm}$ from Er-ions, which did not respond to the resonant radiative transitions ${ }^{2} \mathrm{H}_{11 / 2} \rightarrow{ }^{4} \mathrm{I}_{15 / 2}$ and ${ }^{4} \mathrm{~S}_{3 / 2} \rightarrow{ }^{4} \mathrm{I}_{15 / 2}$ and occurs only in cavity structures.

We report here a coherent upconversion emission at $537 \mathrm{~nm}$ in Er-doped silica microspheres, which is further enhanced by metallic coatings, showing low pump threshold at $976 \mathrm{~nm}$. We will show both cavity configurations created by pure Er-doped silica microsphere and by metallic coated Er-doped silica microsphere, which supported the emission enhancement and wavelength shift.

\section{EXPERIMENTAL SETUP}

The Er-doped silica glasses used in experiment are high-concentration Er-doped silica, which obtained from fibre core part of the commercial fibre HCO 4000. We designed and prepared microsphere cavities based on Er-doped silica glass. We have developed a method to make a microsphere cavity at the end of an Er-doped silica fiber using a thermal melting method. The Er-doped optical fiber is etched in hydrogen fluoride acid (HF) solution in order to dissolve the cladding and leave the naked core part with Er-doped silica (with diameter of about $5 \mu \mathrm{m}$ ). The electrical arc is used for preparation of the Er-doped silica glass microsphere with diameters of 60-120 $\mu \mathrm{m}$. The pump direction is through the fiber tip toward the center of the microsphere. A high resolution spectrometer (MicroSpec2300i) with a slit of $20 \mu \mathrm{m}$, spectral resolution of $0.1 \mathrm{~nm}$, and light power sensitivity of $-90 \mathrm{dBm}$ was used for measuring the emission spectra. The light is collected by an optical fiber with diameter of $1 \mathrm{~mm}$, which allows easy adjustment of the light collection angle.

\section{RESULTS AND DISCUSSION}

Fig.1 shows experimentally observed green emission spectrum from Er-ions doped in the optical fibre HCO 4000, when the pumping power was of $65 \mathrm{~mW}$ at wavelength of $976 \mathrm{~nm}$. The single-mode emission at $537.26 \mathrm{~nm}$ with linewidth of $0.2 \mathrm{~nm}$ obtained. The upconversion emission intensity at $537 \mathrm{~nm}$ from the Er- doped silica fiber is maximal at the perpendicular angle to fiber axis and its distribution is homogeneously around the fiber.

We proposed that random cavity structure was created on fibre by silica glass- air gap polymer coated layer, which can imaged by scanning electron microscope (SEM) S-4800 (see inset of Fig. 1). The spectrum of upconversion emissions from the Er-doped silica fiber has the narrow band of $537 \mathrm{~nm}$, which observed radially from the random microcavity, and the usual fluorescence with a large-width spectrum having two maxima at 523.2 and $547.2 \mathrm{~nm}$. Fig. 2 demonstrates the 


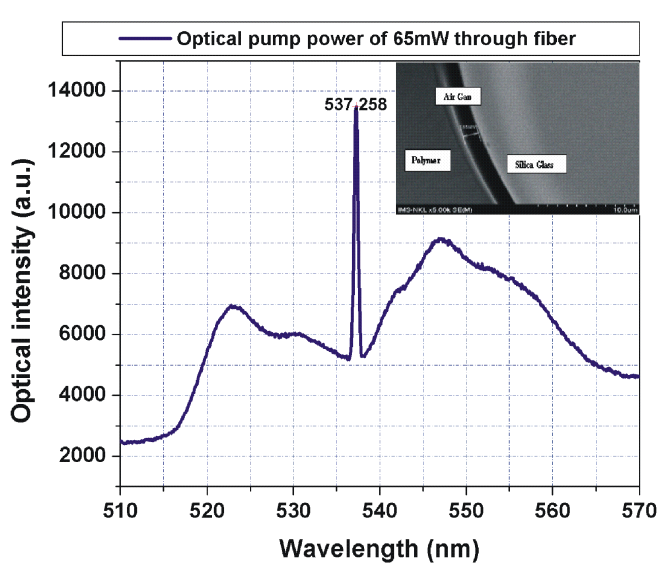

Fig. 1. Spectrum of $537 \mathrm{~nm}$ emission obtained from outside of Er-doped silica fiber. Inset: SEM image of air gap between silica glass and polymer

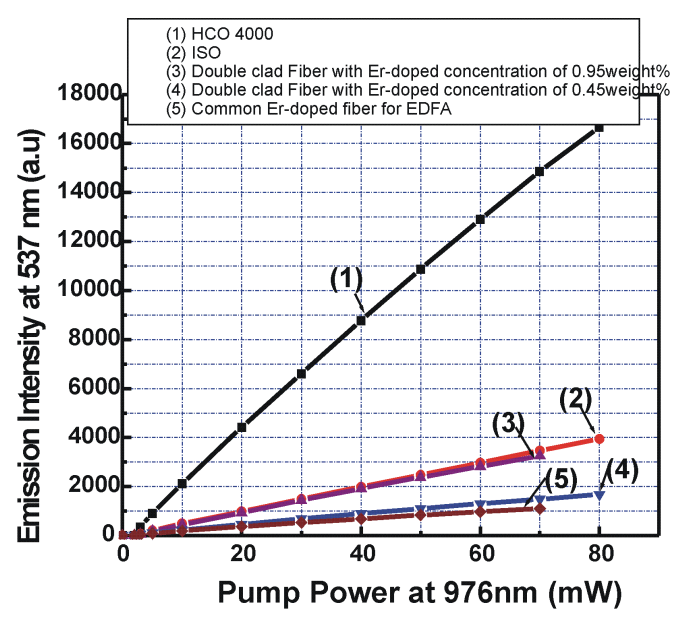

Fig. 2. The $537 \mathrm{~nm}$ narrow emission intensity versus the $976 \mathrm{~nm}$-laser pump power for different kind of Er-doped silica fibers

intensity of $537 \mathrm{~nm}$ narrow line-width emission as a function of the pump intensity at $976 \mathrm{~nm}$ for different fibres. Upon increasing the pumped intensity, the $537 \mathrm{~nm}$ green light intensity stays in the linear lasing regime [11].

It should be noted that the lasing threshold was low (at pumped power of 2-3 $\mathrm{mW}$ for $6 \mathrm{~m}$ long Er-doped fibre) and the laser output remains linear with respect to the optical pump power even at some ten times above threshold. As outlined in Fig. 2, when the Er-ion concentration doping into HCO 4000 is highest in comparison with others, the $537 \mathrm{~nm}$ - emission intensity strongly depends on the Er-concentration in silica glass.

For testing formation of a random cavity that has structure of glass-air gap-polymer cover, we destroyed the air-gap by three ways: (i) removing the coated polymer cover, (ii) destroying air-gap by depressing polymer cover and (iii) covering polymer layer by water and/or alcohol. For the first two cases (without air-gap in the structure of the fibre) the emission at $537 \mathrm{~nm}$ was disappeared, that mean the cavity of laser was absolutely destroyed. In the last case, the $537 \mathrm{~nm}$ emission intensity was decreased by light scattering on the liquid layer. It is remarkable that narrow green emission at wavelength of $537 \mathrm{~nm}$ is insensitively to polarization.

From Er-doped silica microsphere we obtained the same lasing emission in the $537 \mathrm{~nm}$ range with slightly wavelength shift (about $\pm 0.1 \mathrm{~nm}$ ) in comparison with circular form cavity on the fibre. We see here that in the sphere when the narrowband peak appears, the fluorescence bands disappear. This is different from the case of the previous observations of emission from fibers, where the peak appeared as added to the usual fluorescence bands. The green emission intensity of microsphere cavity strongly depended on the measurement direction. It is maximal at the parallel direction and minimal at orthogonal one respect to pump direction, while the emission wavelengths are the same for both cases. Using the model of the coupled photon-atom modes in the cavity [7] we propose that the $537 \mathrm{~nm}$ emission from Er-ions in the silica glasses can appear by following factors: A diode laser operating at $976 \mathrm{~nm}$ pumps the Er-ions from their fundamental 


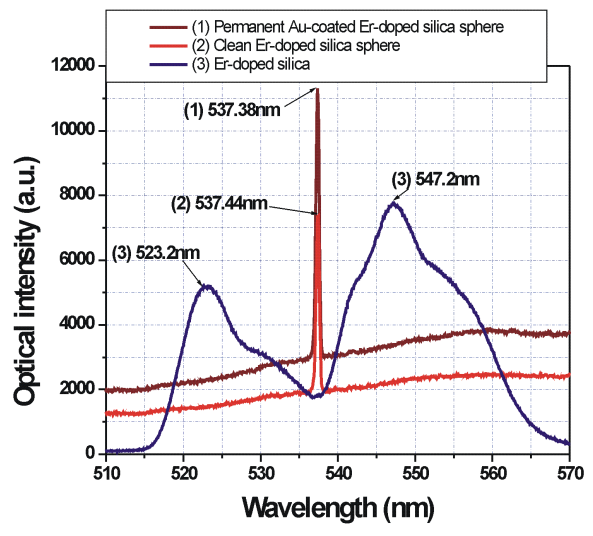

Fig. 3. Spectra of upconversion emission from Er-doped glasses in different surface forms: (1) $50 \mathrm{~nm}$ gold coated hemisphere surface on microsphere, (2) pure surface of silica microsphere, (3) glass bulk. Pump power was of $40 \mathrm{~mW}$

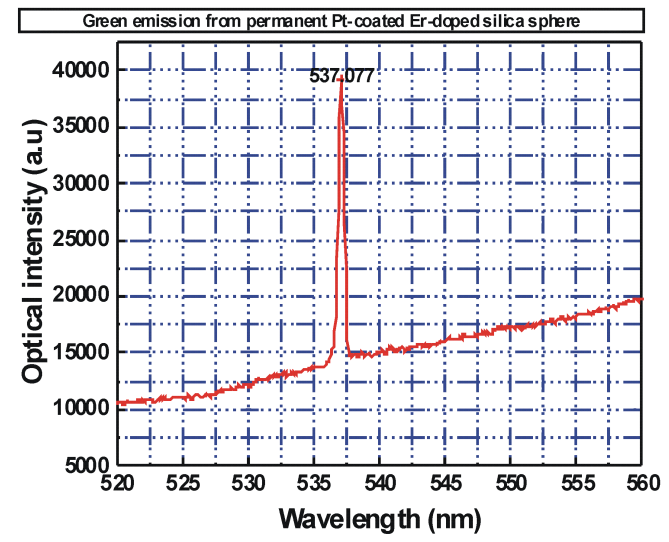

Fig. 4. Spectrum of upconversion emission from Pt-coated on hemisphere of silica microsphere cavity. The Pt-film thickness is $750 \mathrm{~nm}$, optical pump power at $976 \mathrm{~nm}$ wavelength is $2 \mathrm{~mW}$, and the measuring angle is orthogonally to pump direction

level ${ }^{4} \mathrm{I}_{15 / 2}$ to ${ }^{4} \mathrm{I}_{11 / 2}$ and a second photon transfers the excited ion to other level ${ }^{4} \mathrm{~F}_{7 / 2}$. This level decay very rapidly to the levels ${ }^{2} \mathrm{H}_{11 / 2}$ and ${ }^{4} \mathrm{~S}_{3 / 2}$. The splitting of these levels is only some hundreds of $\mathrm{cm}^{-1}$ and the inversion can be achieved between the level ${ }^{4} \mathrm{~S}_{3 / 2}$ and the upper Stark level of the ground state ${ }^{4} \mathrm{I}_{15 / 2}$. In our case, the emission at $537 \mathrm{~nm}$ does not respond to radiative transition between the excited state ${ }^{4} S_{3 / 2}$ and fundamental state ${ }^{4} I_{15 / 2}$, this means that the emitted photon is result of the interaction between the resonant cavity photon and the excited ions on the upper levels ${ }^{2} \mathrm{H}_{11 / 2}$ and ${ }^{4} \mathrm{~S}_{3 / 2}$. We therefore expect that the confined photons can be coupled to excited atom on states ${ }^{2} \mathrm{H}_{11 / 2}$ and ${ }^{4} \mathrm{~S}_{3 / 2}$ such as exciton - polaritons in semiconductors [12,13], and this has influenced to cavity resonant wavelength.

A metallic-assisted microsphere cavity is created by coating metallic layer on the silica glass microsphere. In our case we use vacuum deposition method such as thermal deposition and/or sputtering for coating gold ( $\mathrm{Au})$, aluminum $(\mathrm{Al})$, and platinum $(\mathrm{Pt})$, respectively, on the surface of Er-doped silica glass microspheres. The thicknesses of coating metallic layers changed from $50 \mathrm{~nm}$ to $750 \mathrm{~nm}$. Fig. 3 shows the spectra and intensities of upconversion emissions from different configurations of Er-doped silica glasses. The experiment was carried out under following conditions: a silica glass with Er-ion concentration of $4000 \mathrm{ppm}$, a diameter of microsphere of $100 \mu \mathrm{m}$, an optical pump power at $976 \mathrm{~nm}$-wavelength changed from 0 to $100 \mathrm{~mW}$, the measuring angle of $0-90^{\circ}$ to pump direction. In the case of glass bulk we obtained luminescent emission with large-width spectrum having two peaks at $523.2 \mathrm{~nm}$ and $547.2 \mathrm{~nm}$. In the case of silica glass microsphere for both configurations of clean silica sphere surface and of $50 \mathrm{~nm}$-thickness gold-coated on hemisphere surface, the lasing single modes at wavelengths of $537.44 \mathrm{~nm}$ and $537.38 \mathrm{~nm}$ are observed, but the emission intensity of Au-coated hemisphere surface is significantly increased in comparison with pure silica surface. Thus, we conclude that the $50 \mathrm{~nm}$-Au layer works as 
reflection mirror and it does not exhibit the absorption associated to surface plasmon resonance (SPR) [14].

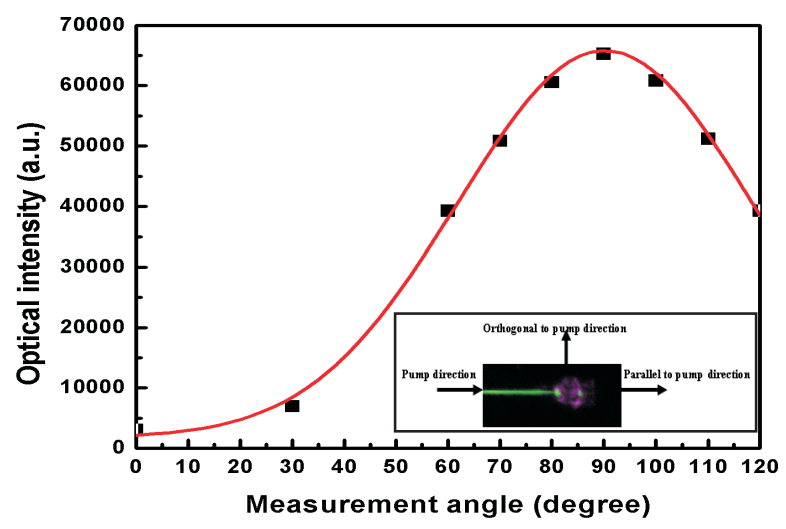

Fig. 5. Emitted intensity distribution versus measurement angle to pump direction for Pt-coated microsphere cavity. The optical pump power at $976 \mathrm{~nm}$-wavelength is $3.5 \mathrm{~mW}$. Inset: Experimental setup for pump and measuring upconversion emission from microsphere cavity

The Pt - layer coated on the hemisphere of Er-doped silica microsphere was developed for study of the role of metallic film on the operation of microsphere cavity. Fig. 4 shows the spectrum and emission intensity in visible range from Er-doped microsphere cavity with $750 \mathrm{~nm}$ thickness Pt-layer coated on hemisphere. The pump optical power at $976 \mathrm{~nm}$ is of $2 \mathrm{~mW}$ and the measurement angle is perpendicular to pump direction. We obtained that the emission intensity of this case is very strongly increased (of 10-20 times) and the emitted wavelength is slightly decreased (on $0.36 \mathrm{~nm}$ ) in comparison with other studied microcavities [15]. The emitted intensity distribution versus measurement angle from metallic-coated sphere cavity is shown in Fig. 5.

An emission distribution by angle to pump direction is coincided with Gaussian law. It is remarkable that green light emission spectrum and intensity are insensitively to change of temperature in the range from $10{ }^{\circ} \mathrm{C}$ to $400^{\circ} \mathrm{C}$. As we known, the surface plasmon coupled emission (SPCE) on Pt-film had resonant wavelength in the range of 520-570 nm [16] and the strong intensity increase and wavelength shift of green light emission from hemispherical Pt-coated cavity may be caused by metal-enhanced fluorescence (MEF).

The Al-coated silica microsphere was made by thermal vacuum deposition of pure Al-metal with Al-layer thickness of $200 \mathrm{~nm}$. The lasing threshold of $537 \mathrm{~nm}$ emission is obtained at $60 \mathrm{nW}$ of pump power. Fig. 6 shows the emission spectrum from Al-coated silica microsphere pumped by $976 \mathrm{~nm}$ laser diode of $60-80 \mathrm{nW}$. The narrow lasing line at $537 \mathrm{~nm}$-wavelenght was appeared on the large spectral noise. 

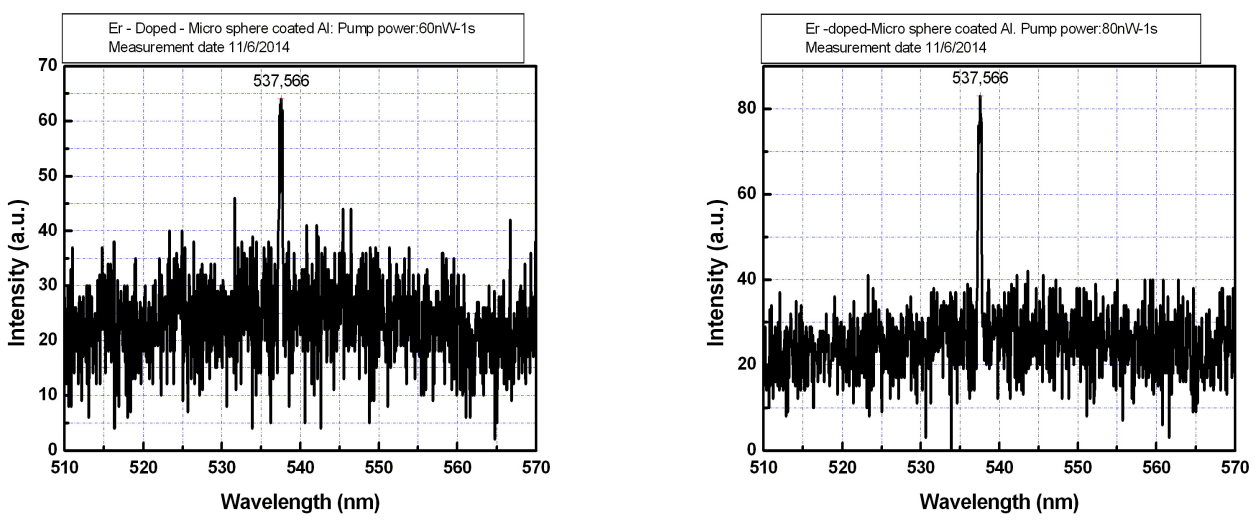

Fig. 6. Spectral lasing intensity of $537 \mathrm{~nm}$-emission from Al-coated silica microsphere at $976 \mathrm{~nm}$-pump power of $60 \mathrm{nW}$ (left) and $80 \mathrm{nW}$ (right)

Fig. 7 presents the characteristic of lasing intensity versus pump power for $\mathrm{Au}, \mathrm{Pt}$, and Al-coated microspheres with the same diameter of $100 \mu \mathrm{m}$. The lasing threshold of Al-coated microsphere was decreased on ten or hundred times in comparison with Pt- and Au-coated spheres.

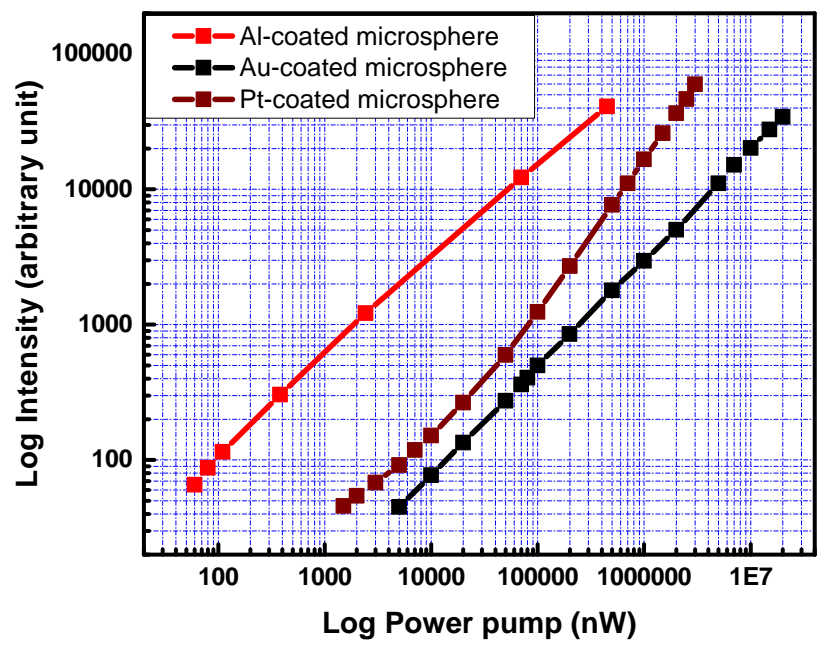

Fig. 7. Characteristics of lasing intensity versus pump power of $\mathrm{Al}, \mathrm{Au}$, and Pt-coated silica microspheres

According to the results presented above, we see that $50 \mathrm{~nm}$-gold layer works as a hemisphere-form mirror supporting for reflection and collection of light emitted by Er-ions in the silica glass, but the Pt- and Al- coated layer simultaneously plays a role of reflection and enhancement of light by SPCE effect. Interpretation of the results is not simple, as the surface 
plasmonic resonant frequencies depend on various factors: the different materials and their sizes, the configurations of metal surfaces and the distributions of optical fields [17-19]. Further work is needed (emission enhancement by surface plasmonic resonance, thermal effect on upconversion emission, degradation of plasmonic enhancement by oxidation...) in order to make a full theory of abnormal lasing emission at $537 \mathrm{~nm}$ from Er-ions in weak-confining cavity as a function of microcavity data and pump.

\section{CONCLUSIONS}

In conclusion, we have observed experimentally for the narrow green light emission at wavelength of $537 \mathrm{~nm}$ from Er-ions doped into silica glasses, which does not respond to any radiative transitions of the erbium ions, in the weak-coupling cavity. This phenomenon is interesting for fundamental investigations, especially for photon-atom interaction at room temperature. The metallic-coated microsphere cavities have emission intensity increase by reflection as mirror and enhancement of light by SPCE effect with a slightly wavelength shift of emitted light in comparison with non-coated silica cavity. The dependence of collected emission intensity on the measurement angle shows a significant potential for optical sensor applications.

\section{ACKNOWLEDGEMENTS}

This work has been done in framework of National Program for Progress in Physics to 2020. The experiments have used the equipment of National Key Laboratory for Electronic Materials and Devices of Institute of Materials Science, Vietnam Academy of Science and Technology.

\section{REFERENCES}

[1] A. Steckl and R. Birkhahn, Applied Physics Letters 73 (12) (1998) 1700-1702.

[2] S. Sergeyev and B. Jaskorzynska, Phys. Rev. B 62 (23) (2000) 15628.

[3] W. Von Klitzing, E. Jahier, R. Long, F. Lissillour, V. Lefevre-Seguin, J. Hare, J. Raimond, and S. Haroche, Journal of Optics B: Quantum and Semiclassical Optics 2 (2) (2000) 204.

[4] H. Sun, S. Dai, S. Xu, L. Wen, L. Hu, and Z. Jiang, Materials Letters 58 (30) (2004) 3948-3951.

[5] O. Toma, IEEE Journal of Quantum Electronics 43 (7) (2007) 519-526.

[6] G. Qin, T. Yamashita, Y. Arai, T. Suzuki, and Y. Ohishi, Optics Communications 279 (2) (2007) 298-302.

[7] M. Lipson and L. Kimerling, Applied Physics Letters 77 (2000) 1150.

[8] E. Schubert, A. Vredenberg, N. Hunt, Y. Wong, P. Becker, J. Poate, D. Jacobson, L. Feldman, and G. Zydzik, Applied Physics Letters 61 (12) (1992) 1381-1383.

[9] K. J. Vahala, Nature 424 (6950) (2003) 839-846.

[10] C.-S. SON, S.-I. KIM, Y.-H. KIM, I.-K. HAN, T. K. YONG, A. WAKAHARA, I.-H. CHOI, and H. CASTANEDA LÖPEZ, Journal of the Korean Physical Society 45 (4) (2004) 955-958.

[11] V. H. Pham, T. C. Do, H. Bui, T. A. Nguyen, and T. V. Nguyen, Advances in Natural Sciences: Nanoscience and Nanotechnology 2 (1) (2011) 015004.

[12] C. Weisbuch, M. Nishioka, A. Ishikawa, and Y. Arakawa, Phys. Rev. Lett. 69 (23) (1992) 3314.

[13] H. Deng, H. Haug, and Y. Yamamoto, Reviews of Modern Physics 82 (2) (2010) 1489.

[14] A. Serrano, O. R. de la Fuente, and M. García, Journal of Applied Physics 108 (7) (2010) 074303.

[15] V. H. Pham, Q. M. Ngo, R. Coisson, et al., Optics Letters 39 (4) (2014) 907-909.

[16] K. Ray, M. H. Chowdhury, and J. R. Lakowicz, Chemical Physics Letters 465 (1) (2008) 92-95.

[17] C. Sauvan, G. Lecamp, P. Lalanne, and J. Hugonin, Optics Express 13 (1) (2005) 245-255.

[18] G. Sun, J. Khurgin, and R. Soref, JOSA B 25 (10) (2008) 1748-1755.

[19] V. H. Nguyen and B. H. Nguyen, Advances in Natural Sciences: Nanoscience and Nanotechnology 3 (3) (2012) 035009. 